\title{
MORDELL-WEIL GROUPS OF GENERIC ABELIAN VARIETIES IN THE UNITARY CASE
}

\author{
ALICE SILVERBERG
}

(Communicated by Larry J. Goldstein)

\begin{abstract}
The generic fibre of a fibre system of polarized abelian varieties with level structure, and with endomorphism structure coming from a CMfield, is defined over the function field of the moduli space for the abelian varieties. We prove that the points on this generic abelian variety which are defined over the function field of the moduli space form a finite group. The methods of proof generalize those of Mordell-Weil groups of generic abelian varieties, Invent. Math. 81 (1985), 71-106, to which this paper is a sequel.
\end{abstract}

Introduction. In this paper we will consider fibre systems of polarized abelian varieties over $\mathbf{C}$ characterized by having a fixed CM-field embedded in their endomorphism algebras. If $V$ is the moduli space for such abelian varieties (with level structure), and $W$ is the fibre variety constructed in [4], then the fibre over the generic point of $V$ is an abelian variety defined over the function field of $V$. We consider the points of this abelian variety which are defined over $\mathbf{C}(V)$. Our result is

THEOREM. If $\operatorname{dim} V>0$, then the Mordell-Weil group of the generic fibre is finite.

Here, $V$ is isomorphic to a product of domains of the form $\{r \times s$ complex matrices $\left.Z \mid 1-Z^{t} \bar{Z}>0\right\}$ modulo the action of a discrete subgroup of a unitary group. The setting for which the theorem holds is described more precisely in $\S 1$.

The analogous theorem was proved for $V$ a noncompact quotient of the complex upper half plane by Shioda (Theorem 5.1 of [5]). When $V$ is a quotient of a Hilbert-Siegel space $H_{s}^{r}$ and $V$ is the moduli space for abelian varieties whose endomorphism algebras contain a fixed totally real field, CM-field, totally indefinite quaterion algebra over a totally real field, or quaternion algebra over a CM-field, the analogous theorem was proved in [6] (see also [7]). The present paper makes use of new methods to remove the restriction on the base variety $V$ in the CM-field case.

I owe many thanks to Goro Shimura for suggesting the problem and giving guidance and advice, and to Robert Indik for the proof of Theorem 6.

Notation. We will write $\operatorname{diag}\left(A_{1}, \ldots, A_{s}\right)$ for the matrix with blocks $A_{1}, \ldots, A_{s}$ on the diagonal, $I(r)$ for the identity matrix in $G L_{r}(\mathbf{Z})$, and ${ }^{t} \gamma$ for the transpose of the matrix $\gamma$.

Received by the editors March 26, 1987.

1980 Mathematics Subject Classification (1985 Revision). Primary 11G10, 11G15; Secondary $14 \mathrm{~K} 22$.

The author was partially supported by an NSF Postdoctoral Fellowship. 
1. Background. In this section we recall the set-up of [2 and 4]. Let $K$ be a totally imaginary quadratic extension of a totally real number field $F$, let $\rho$ be complex conjugation, and let $g=[F: \mathbf{Q}]$. We may view $K$ as embedded in $\mathbf{C}^{g} \cong K \otimes_{\mathbf{Q}} \mathbf{R}$, and for $k=1, \ldots, g$ we let $\tau_{k}$ be the projection of $K \otimes \mathbf{R}$ onto the $k$ th factor. Fix an integer $m>1$ and a matrix $T \in G L_{m}(K)$ with ${ }^{t} T^{\rho}=-T$. Suppose $i\left(T^{\tau_{k}}\right)$ has signature $\left(r_{k}, s_{k}\right)$ for $k=1, \ldots, g$. By renumbering the $\tau_{k}$ 's, and replacing $\tau_{k}$ by $\rho \tau_{k}$ if necessary, we may assume $r_{k} \geq s_{k}$ for all $k$, and $r_{1}=\min _{k} r_{k}$. We must assume $T$ is indefinite, i.e., $r_{1} \neq m$ (otherwise the base variety $V$ is a single point and our theorem clearly fails). For $x=\left(x_{1}, \ldots, x_{g}\right) \in \mathbf{C}^{g}$, let $\Phi(x)=\operatorname{diag}\left(x_{1} I\left(r_{1}\right), \bar{x}_{1} I\left(s_{1}\right), \ldots, x_{g} I\left(r_{g}\right), \bar{x}_{g} I\left(s_{g}\right)\right) \in M_{n}(\mathbf{C})$, where $n=g m$. Fix a lattice $\mathscr{M} \subset K^{m}$ of rank $2 n$ and so that $\operatorname{tr}_{K / Q}\left(\mathscr{M} T^{t} \mathscr{M}^{\rho}\right) \subset \mathbf{Z}$.

We can find $W \in G L_{m}(K)$ so that $W T^{t} W^{\rho}$ is diagonal. Therefore by adjusting the lattice $\mathscr{M}$, we may assume $T$ is diagonal and of the form

$$
T=\alpha \cdot \operatorname{diag}\left(a_{1}, \ldots, a_{m}\right) \text { with } \alpha \in K^{\times}, \alpha^{\rho}=-\alpha, \text { and } a_{1}, \ldots, a_{m} \in F^{\times} .
$$

Let $J_{k}=\operatorname{diag}\left(I\left(r_{k}\right),-I\left(s_{k}\right)\right)$, for $k=1, \ldots, g$. There exist matrices $W_{k} \in G L_{m}(\mathbf{R})$ so that $i W_{k} T^{\tau_{k} t} W_{k}=J_{k}$ for $k=1, \ldots, g$. Further, we can choose $W_{k}$ in the form $P_{k} \cdot D_{k}$ where $D_{k}$ is diagonal and $P_{k}$ is a permutation matrix defining a permutation of order two, $\pi_{k}$, on the set $\{1, \ldots, m\}$. Also we may rearrange $a_{1}, \ldots, a_{m}$ so that $W_{1}$ is diagonal-then $\pi_{1}=1$.

Let $\mathbf{H}=\mathbf{H}(T)=\bigoplus_{k=1}^{g} H\left(r_{k}, s_{k}\right)$ where

$$
H(r, s)=\left\{r \times s \text { matrices } Z \mid I(r)-Z^{t} \bar{Z} \text { is positive hermitian }\right\} .
$$

For $Z=\left(Z_{1}, \ldots, Z_{g}\right) \in \mathbf{H}$, let

$$
X_{k}=\left(\begin{array}{cc}
I\left(r_{k}\right) & Z_{k} \\
t \bar{Z}_{k} & I\left(s_{k}\right)
\end{array}\right) \quad W_{k}=\left(\begin{array}{ccc}
u_{1, k} & \cdots & u_{m, k} \\
\bar{v}_{1, k} & \cdots & \bar{v}_{m, k}
\end{array}\right)
$$

with $u_{j, k} \in \mathbf{C}^{r_{k}}, v_{j, k} \in \mathbf{C}^{s_{k}}$ for $k=1, \ldots, g$ and let

$$
R_{l}(Z, T)={ }^{t}\left({ }^{t} u_{l, 1},{ }^{t} v_{l, 1}, \ldots,{ }^{t} u_{l, g},{ }^{t} v_{l, g}\right) \quad \text { for } l=1, \ldots, m .
$$

For $x=\left(x_{1}, \ldots, x_{m}\right) \in(K \otimes \mathbf{R})^{m}$ let

$$
\eta_{T}(x, Z)=\sum_{l=1}^{m} \Phi\left(x_{l}\right) R_{l}(Z, T) .
$$

Also fix elements $v_{1}, \ldots, v_{s} \in K^{m}$, let $\mathscr{O}=\{a \in K \mid a \mathscr{M} \subset \mathscr{M}\}$, and let $\mathscr{N}=$ $\mathscr{M}+\sum_{j=1}^{s} \mathscr{O} v_{j}$. Let $G=\left\{\left.\gamma \in G L_{m}(K \otimes \mathbf{R})\right|^{t} \gamma^{\rho} T \gamma=T\right\}$, let $G_{K}=G \cap G L_{m}(K)$, and let $\Gamma=G_{K} \cap\left\{\gamma \in M_{m}(K) \mid M_{\gamma}=\mathscr{M}, N(1-\gamma) \subset \mathscr{M}\right\}$. As in [6] we may assume the $v_{j}$ 's have been chosen so that $\Gamma \subset S L_{m}(K)$ and $\Gamma$ has no nonidentity elements of finite order. Let $G(r, s)$ be the unitary group $\left\{\left.\gamma \in G L_{m}(\mathbf{C})\right|^{t} \bar{\gamma} J \gamma=J\right\}$, where $J=\operatorname{diag}(I(r),-I(s))$. Then $G(r, s)$ acts on $\mathbf{H}(r, s)$ : for $Z \in \mathbf{H}(r, s)$, and

$$
\gamma=\left(\begin{array}{ll}
a & b \\
c & d
\end{array}\right) \in G(r, s)
$$

with $a \in M_{r}(\mathbf{C})$ and $d \in M_{s}(\mathbf{C})$, we have $\gamma(Z)=(a Z+b)(c Z+d)^{-1}$. The map $\gamma \rightarrow\left({ }^{t} W_{1}^{-1} \gamma^{\tau_{1} t} W_{1}, \ldots,{ }^{t} W_{g}^{-1} \gamma^{\tau_{g} t} W_{g}\right)$ is an isomorphism from $G$ to $\bigoplus_{k=1}^{g} G\left(r_{k}, s_{k}\right)$, and defines an action of $G$ on $\mathbf{H}$. 
For $Z \in \mathbf{H}$, let $A_{Z}=C^{n} / \eta_{T}(\mathscr{M}, Z)$, let $C_{Z}$ be the polarization of $A_{Z}$ defined by $T$ (see (11) of [2]), let $\theta_{Z}=\Phi$, and let $t_{j}(Z)=\eta_{T}\left(v_{j}, Z\right)$. Write $Q_{Z}=\left(A_{Z}, C_{Z}, \theta_{Z}, t_{1}(Z), \ldots, t_{s}(Z)\right)$. Then $Q_{Z}$ is a polarized abelian variety of type $\left(K, \Phi, \rho, T, \mathscr{M}, v_{1}, \ldots, v_{s}\right)$ (see Definition 1.2 of [6]). By 3.6 of [4] there is a map $\phi: \mathbf{H} \rightarrow \mathbf{P}^{M}$ inducing an embedding of $\mathbf{H} / \Gamma$ into a projective space $\mathbf{P}^{M}$ so that $V=\phi(\mathbf{H})$ is a nonsingular variety. Further, by 3.8 of [4], there is a fibre system $(V, W, \ldots)$ of abelian varieties so that for $Z \in \mathbf{H}$ and $u=\phi(Z)$, the fibre $Q_{u}=\left(A_{u}, \ldots\right)$ is isomorphic to $Q_{Z}$. The variety $W$ is analytically isomorphic to $\left(\mathbf{H} \times \mathbf{C}^{n}\right) /(\Gamma \times \mathscr{M})$, where the group law and action are defined as in 3.14 of [4] or 1.4 of [6]. The fibre over a generic point of $V$ is an abelian variety defined over the function field $\mathbf{C}(V)$ of $V$.

PROPOSITION 1. The group of points over $\mathrm{C}(V)$ of the generic fibre is isomorphic to the group of holomorphic sections from $V$ to $W$.

PROOF. Corollary 2.2 of [6].

THEOREM I. In the situation described above, the Mordell-Weil group over $\mathbf{C}(V)$ of the generic fibre is finite.

When $m=2$, the theorem was proved for $\operatorname{dim} V>1$ in case $\mathrm{II}_{c}$ of the Main Theorem of [6] (see $\S 5$ of [6]), for $\operatorname{dim} V=1$ and $V$ compact in Case $\mathrm{I}_{c}$ of the Main Theorem of [6] (see $\S 4.3$ of [6]), and for $\operatorname{dim} V=1$ and $V$ not compact in Shioda's Theorem 5.1 of [5]. In what follows, we will assume $m \geq 3$.

2. Reduction to lower dimensions. Our method of proof is to pull back the fibre system to fibre systems of the same type, but with $m=2$. We can then use the results for $m=2$ to give a dense set of points in $V$ whose images under sections are points of finite order, and whose fibres have CM by $K^{m}$. In $\S 3$ we show this suffices to prove Theorem I.

The method of proof here differs from $\S 6$ of [6] in one significant way: in $\S 6.4$ of [6], we pulled back the fibre system to only one fibre system over a lower dimensional base. That technique no longer suffices - it is now sometimes necessary to choose as many as $r_{1} \cdot s_{1}$ different pullbacks, as can be seen by examining the case $i T=$ $\operatorname{diag}(1,1,-1), K=\mathbf{Q}(i)$.

Write $\mathbf{0}$ for the element of $\mathbf{H}$ with zero in every entry.

THEOREM 2. Suppose $h: \mathbf{H} \rightarrow \mathbf{C}^{n}$ is a holomorphic map which induces a section from $V$ to $W$. Then for every $\beta \in G_{K}, h(\beta(0))$ gives a point of finite order in $A_{\beta(0)}$, and for some $\theta_{\beta}$ and $\Phi^{\prime},\left(A_{\beta(0)}, C_{\beta(0)}, \theta_{\beta}\right)$ is of type $\left(K^{m}, \Phi^{\prime}\right)$.

PROOF. Fix positive integers: $i \leq r_{1} ; j \leq s_{1}$. Let $T_{i j}=\alpha \cdot \operatorname{diag}\left(a_{i}, a_{r_{1}+j}\right)$. For $1 \leq k \leq g$ let $\left(r_{k}^{\prime}, s_{k}^{\prime}\right)$ be the signature of $i T_{i j}^{\tau_{k}}$. Let $M_{k}=\pi_{k}(i), N_{k}=\pi_{k}\left(r_{1}+j\right)$, and write $F_{k}(a, b)$ for the $r_{k} \times s_{k}$ matrix with one in position $(a, b)$ and zeros elsewhere. Define a map $\varepsilon\left(=\varepsilon_{i j}\right)$ from $\mathbf{H}\left(T_{i j}\right)=\bigoplus_{k=1}^{g} H\left(r_{k}^{\prime}, s_{k}^{\prime}\right)$ to $\mathbf{H}$ by $\varepsilon\left(z_{1}, \ldots, z_{g}\right)=$ $\left(Z_{1}, \ldots, Z_{g}\right)$ with

$$
Z_{k}= \begin{cases}z_{k} \cdot F_{k}\left(M_{k}, N_{k}-r_{k}\right) & \text { if } M_{k} \leq r_{k} \text { and } N_{k}>r_{k} \\ z_{k} \cdot F_{k}\left(N_{k}, M_{k}-r_{k}\right) & \text { if } M_{k}>r_{k} \text { and } N_{k} \leq r_{k} \\ 0 & \text { otherwise }\end{cases}
$$

Note. Since $\left(r_{1}^{\prime}, s_{1}^{\prime}\right)=(1,1)$, we have $\operatorname{dim} \mathbf{H}\left(T_{i j}\right)>0$. 
For $l=1, \ldots, m$ define projection maps

$$
p_{l}\left(x_{1}, \ldots, x_{m g}\right)=\left(x_{l}, x_{\pi_{2}(l)+m}, \ldots, x_{\pi_{g}(l)+(g-1) m}\right),
$$

and let $p_{i j}=p_{i} \oplus p_{r_{1}+j}$. Observe that $p_{i j}\left(\eta_{T}\left(\mathscr{O}_{K}^{m}, \varepsilon(z)\right)\right)=\eta_{T_{i j}}\left(\mathscr{O}_{K}^{2}, z\right)$. Also, let $T_{l}=\alpha a_{l}$ and observe that $p_{l}\left(\eta_{T}\left(\mathscr{O}_{K}^{m}, \varepsilon(z)\right)\right)=\eta_{T_{l}}\left(\mathscr{O}_{K}, 0\right)$, for $l=1, \ldots, m$ (here, $\left.0 \in \mathbf{H}\left(T_{l}\right)\right)$. Write $\left(A_{Z}^{\prime}, C_{Z}^{\prime}, \theta_{Z}^{\prime}\right)=P_{Z}^{\prime}$ for the abelian variety determined by $Z, T, \mathscr{O}_{K}^{m}$ (as in Theorem 1 of [2]). Then for $z \in \mathbf{H}\left(T_{i j}\right)$,

$$
A_{\varepsilon(z)}^{\prime} \cong \mathbf{C}^{n} / \eta_{T}\left(\mathscr{O}_{K}^{m}, \varepsilon(z)\right) \cong \mathbf{C}^{2 g} / \eta_{T_{i j}}\left(\mathscr{O}_{K}^{2}, z\right) \oplus \bigoplus_{\substack{l=1 \\ l \neq i, r_{1}+j}}^{m} \mathbf{C}^{g} / \eta_{T_{l}}\left(\mathscr{O}_{K}, 0\right)
$$

Fix $\beta \in G_{K}$. By 2.8 of [2] (see also $\S 6.3$ of $\left.[6]\right),\left(A_{\beta(Z)}, C_{\beta(Z)}, \theta_{\beta(Z)}\right)$ is isogenous to $P_{Z}^{\prime}$ for $Z \in \mathbf{H}$. For $z \in \mathbf{H}\left(T_{i j}\right)$, write $\lambda_{z}$ for the isogeny from $A_{\beta \circ \varepsilon(z)}$ to $A_{\varepsilon(z)}^{\prime}$, and $\Lambda_{z}$ for the associated map from $\mathbf{C}^{n}$ to $\mathbf{C}^{n}$. As in $\S 6$ of [6], the map

$$
h_{i j}(z)=p_{i j} \circ \Lambda_{z} \circ h \circ \beta \circ \varepsilon(z)
$$

from $\mathbf{H}\left(T_{i j}\right)$ to $\mathbf{C}^{2 g}$ is holomorphic. We can construct a fibre system of polarized abelian varieties of type $\left(K, \Phi_{i j}, \rho, T_{i j}, \mathscr{O}_{K}^{2}\right.$, representatives for $\left.(1 / M) \mathscr{O}_{K}^{m} / \mathscr{O}_{K}^{m}\right)$ with base $\mathbf{H}\left(T_{i j}\right) / \Delta$ for $\Delta$ the principal congruence subgroup of level $M$ in $\{\delta \in$ $\left.\left.S L_{2}\left(\mathscr{O}_{K}\right)\right|^{t} \delta^{\rho} T_{i j} \delta=T_{i j}\right\}$, for a suitable level $M$ so that $\Delta$ embeds in $\beta^{-1} \Gamma \beta$ in a natural way. Here, $\Phi_{i j}$ is defined by $\left\{\left(r_{k}^{\prime}, s_{k}^{\prime}\right)\right\}_{k=1}^{g}$. By Theorem 5.1 of [5] and Cases $\mathrm{I}_{c}$ and $\mathrm{II}_{c}$ of the Main Theorem of [6], $h_{i j}$ is a section of finite order for this new fibre system. Thus, for every $z \in \mathbf{H}\left(T_{i j}\right), \beta \in G_{K}, 1 \leq i \leq r_{1}, 1 \leq j \leq s_{1}$, $h_{i j}(z)$ is a point of finite order in $\mathbf{C}^{2 g} / \eta_{T_{i j}}\left(\mathscr{O}_{K}^{2}, z\right)$.

Now let $z=0$ in $\mathbf{H}\left(T_{i j}\right)$ (and then $\varepsilon(z)=0$ in $\mathbf{H}$ ). Let $E_{l}=\mathbf{C}^{g} / \eta_{T_{l}}\left(\mathscr{O}_{K}, 0\right)$ for $1 \leq l \leq m$. $E_{l}$ has CM by $\left(K, \Phi_{l}\right)$, where for $x \in K \otimes \mathbf{R}, \Phi_{l}(x)=\operatorname{diag}\left(x^{\delta_{1}}, \ldots, x^{\delta_{g}}\right)$ with

$$
\delta_{k}= \begin{cases}\tau_{k} & \text { if } \pi_{k}(l) \leq r_{l} \\ \rho \tau_{k} & \text { if } \pi_{k}(l)>r_{l}\end{cases}
$$

Then $A_{0}^{\prime} \cong \bigoplus_{l=1}^{m} E_{l}$, and $\left\{\Phi_{l}\right\}_{l=1}^{m}$ defines a map $\Phi^{\prime}: K^{m} \rightarrow M_{n}(\mathbf{C})$ and an embed$\operatorname{ding} \tilde{\theta}_{0}: K^{m} \rightarrow \operatorname{End}\left(A_{0}^{\prime}\right) \otimes \mathbf{Q}$. For $\beta \in G_{K}$, the map $\lambda_{0}$ defines an isogeny from $\left(A_{\beta(0)}, C_{\beta(0)}, \lambda_{0}^{-1} \tilde{\theta}_{0} \lambda_{0}\right)$ to $\left(A_{0}^{\prime}, C_{0}^{\prime}, \tilde{\theta}_{0}\right)$. For every $i$ and $j, h_{i j}(0)$ gives a point of finite order in $E_{i} \times E_{r_{1}+j}$. Thus $\Lambda_{o} h(\beta(0))$ has finite order in $A_{0}^{\prime}$. Consequently, for every $\beta \in G_{K}, h(\beta(0))$ has finite order in $A_{\beta(0)}$.

3. Conclusion of proof of Theorem I. By Theorem 5.3 of [4], the fibre system $(V, W, \ldots)$ is defined over a number field $k_{\Omega}$ so that for every $u \in V, k_{\Omega}(u)$ is the field of moduli of the fibre $Q_{u}$. For $\beta \in G_{K}$, let $\tilde{\beta}=\phi(\beta(0)) \in V$. Then $A_{\tilde{\beta}} \cong A_{\beta(0)}$.

PROPOSITION 3. Suppose $L$ is a subfield of $\mathbf{C}$ which is finitely generated over $\mathbf{Q}$ and contains $k_{\Omega}$. There is a constant $B_{L}$ so that for every $\beta \in G_{K},\left|A_{\tilde{\beta}}(L(\tilde{\beta}))_{\text {tors }}\right| \leq$ $B_{L}$.

PROOF. This follows immediately from Theorem 2 above, and Theorem A of $\S 7.1$ of [6] (see also Theorem 2 of [7]). 


\section{Proposition 4. All sections are torsion.}

PROOF (SEE $\S 7.8$ OF [6]). Fix a section and chocse $L$ to be a field over which the section is defined. The result follows from Proposition 3 and the fact that $\left\{\tilde{\beta} \mid \beta \in G_{K}\right\}$ is dense in $V$.

Theorem I now follows, either by a Mordell-Weil theorem for abelian varieties over function fields, or by bounding the number of holomorphic sections of finite order. Such a bound is given by Corollary 2.7 of [6], which depends on the following lemma (Lemma 2.4 of [6] when the base variety was a quotient of a Hilbert-Siegel space).

LEMMA 5. There is an element $\gamma \in \Gamma$ so that $\operatorname{det}(1-\gamma) \neq 0$.

We first take a more general setting. Suppose now that $K$ is any number field. Write $K_{\mathbf{A}}$ for the finite adèles of $K$, and write $\mathscr{O}_{v}$ for the ring of integers of the completion $K_{v}$. Suppose $H \subset G L_{m}(\mathbf{C})$ is a linear algebraic group defined over $K$. We have the usual topological groups $H_{K}, H_{\mathbf{A}}\left(=H_{K_{\mathbf{A}}}\right), H_{v}\left(=H_{K_{v}}\right), H\left(\mathscr{O}_{v}\right)$ $\left(=H_{\mathscr{O}_{v}}\right)$. For us, $H$ will be $G_{K} \cap S L_{m}(K)$, which satisfies the following two properties:

Strong approximation. $H_{K}$ is dense in $H_{A}$.

Local property. For infinitely many primes $w$ of $K$, there is an element $\gamma_{w} \in$ $H\left(\mathscr{O}_{w}\right)$ so that $\operatorname{det}\left(1-\gamma_{w}\right) \neq 0$.

Let $U=\prod_{v} M_{m}\left(\mathscr{O}_{v}\right)$, the product over the finite primes of $K$.

THEOREM 6. Suppose $H$ as above is a group with the strong approximation and local properties. Suppose $N \in \mathbf{Z}^{+}$and $\Gamma_{N}=H_{K} \cap(1+N U)$. Then there is an element $\gamma \in \Gamma_{N}$ with $\operatorname{det}(1-\gamma) \neq 0$.

PROOF. $H_{\mathbf{A}} \cap(1+N U)=\prod_{v \nmid N} H\left(\mathscr{O}_{v}\right) \cdot \prod_{v \mid N}\left(H_{v} \cap\left(1+N M_{n}\left(\mathscr{O}_{v}\right)\right)\right)$. By the local property, we can find a prime $w$ with $w \nmid N$, and $\gamma_{w} \in H\left(\mathscr{O}_{w}\right)$ with $\operatorname{det}\left(1-\gamma_{w}\right) \neq 0$. Let $U_{w}=\left\{\delta \in H\left(\mathscr{O}_{w}\right) \mid \operatorname{det}(1-\delta) \neq 0\right\}$ and let $V=U_{w} \cdot \prod_{v \neq w}\left(H_{v} \cap\left(1+N M_{m}\left(\mathscr{O}_{v}\right)\right)\right)$, a nonempty open subset of $H_{\mathbf{A}}$. By strong approximation, there is an element $\gamma \in V \cap H_{K} \subset \Gamma_{N}$. By the choice of $V, \operatorname{det}(1-\gamma) \neq 0$.

PROOF OF LEMMA 5. Let $H=G_{K} \cap S L_{m}(K)$. Choose positive integers $c, d$, and $R$ so that $d \mathscr{O}_{K}^{m} \subset c \mathscr{M} \subset \mathscr{O}_{K}^{m}$ and $R \mathscr{N} \subset \mathscr{M}$. Then $\Gamma_{R d} \subset \Gamma$, so it suffices to prove the lemma for $\Gamma_{R d}$. $H$ has strong approximation by [3 or 1], so by Theorem 6 it suffices to show $H$ has the local property. If $m$ is even, choose any $w$ with $w+2 N$, and let $\gamma_{w}=-1$. If $m$ is odd, choose $\alpha \in K$ so that $\alpha^{2} \notin F$. Let $\beta=\alpha / \alpha^{\rho}$, and choose $w$ so that $\beta$ is a unit in $\mathscr{O}_{w}$. Let $\gamma_{w}=\operatorname{diag}\left(\beta, \beta, \beta^{-2},-1, \ldots,-1\right)$.

\section{REFERENCES}

1. M. Kneser, Starke Approximation in algebraischen Gruppen. I, J. Reine Angew. Math. 218 (1965), 190-203.

2. G. Shimura, On analytic families of polarized abelian varieties and automorphic functions, Ann. of Math. 78 (1963), 149-192.

3. _ Arithmetic of unitary groups, Ann. of Math. 79 (1964), 369-409.

4. __ Moduli and fibre systems of abelian varieties, Ann. of Math. 83 (1966), 294-338. 
5. T. Shioda, On elliptic modular surfaces, J. Math. Soc. Japan 24 (1972), 20-59.

6. A. Silverberg, Mordell-Weil groups of generic abelian varieties, Invent. Math. 81 (1985), 71-106.

7. __ Finiteness of Mordell-Weil groups of generic abelian varieties, Bull. Amer. Math. Soc. 12 (1985), 131-133.

Department of Mathematics, Ohio State University, 231 W. 18 Avenue, COLUMBUS, OHIO 43210 